\title{
The Role of Hepatitis C Virus in Parkinson's Disease
}

\author{
Fatema Abdulla ${ }^{1}$, Heidi Jalloh-Vos ${ }^{2}$ and Maheeba Abdulla ${ }^{3 *}$ \\ ${ }^{1}$ Clinical Neuroscience, Kingdom of Bahrain \\ ${ }^{2}$ Medical Research Council (MRC), Sierra Leone
}

${ }^{3}$ Department of Internal Medicine, Kingdom of Bahrain

*Corresponding author: Maheeba Abdulla, Department of Internal Medicine, Salmaniya Medical Complex, Kingdom of Bahrain

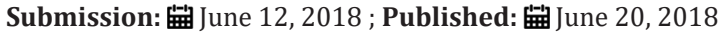

\begin{abstract}
This article summarizes findings of a review of recent articles related to the possible associations between (viral) hepatitis and Parkinson's disease. There is growing evidence for an association between Hepatitis $\mathrm{C}$ and Parkinson's disease, while there is conflicting evidence for such an association between Hepatitis B and Parkinson's disease. More research is needed into the causes of Parkinson's disease, including its relation to Hepatitis B and C. Implications for clinical practice include regular neurological evaluation of HCV positive patients.
\end{abstract}

Keywords: Parkinson's disease; Hepatitis C; Risk factor; Hepatitis B

Abbreviations: Hepatitis C Virus (HCV), Parkinson's Disease (PD), Confidence Interval (CI), Hepatitis Virus (HBV)

\section{Introduction}

Parkinson's disease (PD) is the most common neurodegenerative disorder after Alzheimer's disease. The characteristic motor features (resting tremor, bradykinesia and muscular rigidity) are associated with Lewy bodies and the loss of dopaminergic neurons in the substantia nigra. The non-motor features like cognitive changes, sleep disorders and depression indicate damage in non-dopaminergic areas [1,2]. Treatment of PD is focused is symptomatic with a focus on improving the quality of the patient's life, as no treatment has been found so far that can slow down or stop the neurodegeneration. Recent publications have contributed to the growing body of evidence for associations between Hepatitis C - and possibly also Hepatitis B- and Parkinson's disease [3]. This article summarizes findings of a review of recent articles related to the possible associations between (viral) hepatitis and Parkinson's disease. Pub Med was searched for articles containing both hepatitis and Parkinson's disease in the title and/or abstract. This resulted in 51 articles, of which 17 were found to relevant after reading through. Additional articles were found through references in relevant articles.

\section{Risk Factors for Parkinson's disease}

While the cause of Parkinson's disease remains unknown, it appears to be due to a complex of environmental and genetic factors that affect many basic processes at the cell level [1]. Risk factors include age with a peak after 80 years, ethnicity with highest incidence in the USA in Hispanic whites, geographic region with PD more common in Europe and the Americas and male gender. Age is the greatest risk factor for PD. A whole range of environmental exposures have been found to increase the risk for PD (including pesticides, rural living, $\beta$-blocker use, agricultural occupation, and well-water drinking) or to decrease the risk for PD (tobacco smoking, coffee drinking, NSAID use, calcium channel blocker use, alcohol consumption). Genetic factors include several gene mutations associated positively or negatively with PD, including monogenic forms of PD [1]. Other risk factors include elevated cholesterol, high caloric intake and increased body mass index [2]. Post-infection states and oxidative stress have also been mentioned as risk factors [2].

\section{Hepatitis C Virus Effects on the Neural system}

Blood-brain barrier becomes more permeable with age for viral and other proteins, while with increasing age the immune system also becomes more compromised thus less able to fight viral and other infections $[4,5]$. The potential role of viruses in PD has been described before; especially for Herpes Simplex Virus 1 (HSV-I) and Influenza A virus, but also for measles and more recently for Hepatitis C virus [6]. HSV-I and Influenza A viruses are able to infect the central nervous system, especially in older people, where 
their viral transcripts and protein may cause neuro-inflammation, autophagy and synaptic disruption. These three processes have important parallels with pathology in PD. For example, in PD there is also synaptic dysfunction, while disturbance of the autophagy process results in neurodegeneration [6]. Neurotropic viruses, i.e. viruses that are able to enter the nervous system, can induce encephalopathies which among other effects, may result in Parkinsonism. Influenza, Coxsackie, Japanese encephalitis B, St. Louis, West Nile and HIV viruses are known to cause Parkinsonism [7].

Hepatitis C virus belongs to the Flavivirus family, wellknown for its neurotropic capacities as shown for example in yellow fever and dengue. There is evidence of invasion and even replication of HCV in the human brain. This might explain the cognitive dysfunction, depression and fatigue that are seen more often in hepatitis C than in other hepatic conditions [8]. Proton magnetic-resonance spectroscopy showed elevations of choline/ creatinine ratios in basal ganglia and white matter of HCV patients as compared to health volunteers and HBV patients, which could not be explained otherwise than through HCV itself affecting the brain [9]. In HCV-positive patients with fatigue and cognitive dysfunction indications were found for changes in serotonergic and dopaminergic neurotransmission in single photon emission computerised tomography (SPECT) investigations [10].

HCV negative-strand RNA sequences, which are evidence of viral replication, were found in autopsy brain tissue of HCVinfected patients [11]. HCV has been shown to infect macrophages, microglia, and also some astrocytes in the brain [12], with increased expression of pro-inflammatory cytokines in HCV-positive patients, especially in HCV-infected brain macrophages and microglia cells [13]. A recent article shows viral octapeptides from HCV and other viruses matching with neurogeneration-associated proteins at a level higher than stochastic expectations [14]. This includes many common sequences with single amino acid repeats, and most of these shared octapeptides are part of antigenic determinants that were found in other studies. Despite all indications at the cellular level, until recently there has not been much epidemiological support for the possible association between HCV and PD.

\section{Recent Epidemiological Studies}

A recent systematic review and meta-analysis into the risk of Parkinson's disease in patients with hepatitis $\mathrm{C}$ infection found 5 relevant studies: 3 retrospective cohort studies, 1 cross-sectional study and 1 case-control study [3]. The pooled Odds Ratio of these studies is 1.35 (95\% confidence interval: 1.19-1.52), which thus shows an increased risk of Parkinson's disease among patients with chronic hepatitis C infection. This meta-analysis had an insignificant statistical heterogeneity $(I 2=3 \%)$. The limitations included possible positive publication bias although not confirmed by Egger's regression test $(\mathrm{p}=0.56)$, limited accuracy of diagnosis in the primary coding-based studies and wide variety of confounder adjustment among the studies.
The first included study was a retrospective nationwide population-based cohort study in Taiwan where chronic hepatitis C virus infection cases $(n=10,286)$ and randomly selected age, sex and index year matched comparators without hepatitis $\mathrm{C}$ infection $(n=199,868)$ were taken from the national health insurance database from 1996 to 2011. Selection was based on diagnostic codes for HCV infection and Parkinson's disease. This resulted in crude hazard ratios for developing PD of 0.66 (95\% CI 0.55-0.80) for HBV, 2.50 (95\% CI 2.07-3.02) for HCV and 1.28 (95\% CI 0.88-1.85) for HBV-HCV co-infection. After extensive confounder adjustment (age, sex, index year and a whole range of comorbidities) the association between HCV and PD remained statistically significant with an adjusted hazard ratio of 1.29 (95\% CI 1.06-1.56) [15].

The second included study was a cross-sectional study which obtained its participants $(n=62,276)$ from a community-based integrated screening program in the north of Taiwan from 20002004. HCV diagnosis was based on finding anti-HCV antibodies during the survey, while PD diagnosis was based on finding signs and symptoms of Parkinson's disease during the survey or history of PD in the participant's medical records. The crude odds ratios for having PD were 0.62 (95\% CI 0.48-0.81) for Hepatitis B virus and 1.91 (95\% CI 1.48-2.47) for Hepatitis C virus. After controlling for confounders (smoking, metabolic syndrome, age, sex, education) the association between $\mathrm{HCV}$ and PD remained statistically significant with an odds ratio of 1.39 (95\% CI 1.07-1.80), but was not significant anymore between HBV and PD [16].

The third included study was a hospital-based case-control study in Korea which included Parkinson's disease cases at study hospitals $(n=1,558)$ and sex and age matched randomly selected controls without PD who were seen in the same hospitals for an annual health check-up $(n=1,558)$. HCV diagnosis was based on finding anti-HCV antibodies, while PD diagnosis was done by study hospital physicians as part of routine care. HCV was significantly higher in the PD group than in the control group $(p=0.041)$ for overall age groups, with a significant association $(p=0.011)$ remaining for the group older than 70 years, but no significant association $(\mathrm{p}=0.781)$ for younger age groups. There was no significant association between HBV (HBsAg positive) and PD for overall age groups $(p=0.237)$, although there was an apparent protective effect of HBV for the group older than 70 years (odds ratio $0.47-95 \%$ CI $0.25-0.89, \mathrm{p}=0.019$ ) [17].

The fourth included study was a retrospective cohort (19992011) from the English National Hospitals database with chronic hepatitis $C$ virus cases $(n=48,428)$ and comparators with minor medical/surgical conditions $(n=6,132,124)$. Selection was based on diagnostic codes for HCV infection and Parkinson's disease. Standardization was applied to control for confounders (sex, first admission calendar year for exposure/reference condition, residential area, socioeconomic status). The resulting standardized rate ratio of PD following HBV was 1.76 (95\% CI 1.28-2.37) and following HCV it was 1.51 (95\% CI 1.18-1.90), with no significant 
associations between PD and autoimmune hepatitis, chronic active hepatitis and HIV. The significant associations of PD with HBV (RR 1.82 95\% CI 1.29-2.50) and HCV (RR 1.43 95\% CI 1.09-1.84) remained after excluding episodes of care for PD that occurred within 1 year after the exposure conditions [18].

The fifth included study is a retrospective cohort (2005-2010) from the Medicare database for disabled and elderly (65 years and older) in the USA. A 5\% random sample was drawn from the database and chronic hepatitis $C$ virus cases $(n=6,040)$ and comparators $(n=1,228,849)$ were identified based on the presence (or absence) of a diagnostic code for HCV infection. There was a significant difference $(\mathrm{p}<0.001)$ in PD prevalence between the HCV cases $(0.8 \%)$ and the non-HCV comparators $(1.3 \%)$, however this difference disappeared after matching for potential confounders (age, gender, race, medicare eligibility status). Among the Medicare population there was therefore no association between HCV and PD [19].

\section{Discussion}

While there is growing evidence for an association between Hepatitis C and Parkinson's disease, the association between Hepatitis B and Parkinson's Disease is not very clear. This last association is also less likely because Hepatitis B is from the Hepadnavirus family, which is a different family than the Flavivirus family, to which Hepatitis $\mathrm{C}$ belongs. There is no evidence for neuroinvasion by the Hepatitis B or by other viruses of the Hepadnavirus family. Hepatitis B is not toxic for dopaminergic neurons [16], while it also does not result in elevate choline/creatine ratio's as were seen in HCV [9]. Furthermore, while there are several medications with potential effectiveness against both Hepatitis $C$ virus infection and Parkinson's disease, this has not been reported about Hepatitis B. These medications include Amantadine [20], Coumarin derivatives [21], anti-malarial drugs with a chloroquinoline scaffold [22], and the anti-oxidant mitoquinone (MitoQ) [23].

The two main options for HCV disease to cause Parkinson(ism) appear to be direct HCV infection of the brain or Parkinsonism induced by interferon treatment. Antiviral medications used in hepatitis B and/ or C, especially different interferon types but also ribavirin and cyclosporin [24-33] have been shown to cause Parkinsonism. Unfortunately, all five described epidemiological studies into the relation between Hepatitis C and Parkinson's disease did not control for interferon as a possible confounder. This is not likely to change the results of the Taiwan communitybased study that took place from 2000 to 2004 as interferon was only being reimbursed from 2004 in Taiwan and the interferon treatment rates were low (10-13\%). However, a further analysis of the Taiwan nationwide study showed much higher PD incidence rates for the interferon group (248.75 per 100,000 person-years, relative rate 1.55 - 95\% CI 1.17-2.06) than for the non-interferon group (30.21 per 100,000 person-years, relative rate $0.5-95 \% \mathrm{CI}$ $0.12-2.00)$. For the non-interferon group this was lower than the PD incidence rate in the average-risk Taiwan population (79.75 per
100,000 person-years) [34]. With the advance of accessibility and affordability of direct-acting antivirals (DAAs), for example through production of generic formulations, the possible confounding by interferon will soon be a problem of the past $[35,36]$. Differences between geographic areas regarding PD and HCV prevalence and their risk factors could explain the difference between the discussed epidemiological studies.

The studies discussed offer several possible implications for clinical practice. HCV-positive patients will need regular neurological evaluation to allow for early detection of PD [36]. Optimal treatment of HCV infections should take into account extra hepatic, including brain, manifestations of HCV infection, and thus HCV treatment should be able to cross the blood-brain barrier [13]. Alternatives to interferon like DAAs could be considered to prevent parkinsonism [35,36].

Development of PD drugs can build on new perspectives on the underlying pathways to the association between HCV and PD. This could include drugs targeting epitopes that have shared octapeptides [14] or drugs that are able to decrease or even prevent inflammatory processes like immunomodulatory drugs and therapeutic immunization [37].

Further understanding of the possible causal pathways between Hepatitis C virus and Parkinson's disease will likely contribute further to future development of treatment options that will be able to stop or slow down the neuro degenerative processes.

\section{Conclusion}

There is growing evidence for an association between Hepatitis $\mathrm{C}$ and Parkinson's disease, while there is conflicting evidence for such an association between Hepatitis B and Parkinson's disease. More research is needed into the causes of Parkinson's disease, including its relation to Hepatitis B and C. Implications for clinical practice include regular neurological evaluation of HCV positive patients.

\section{References}

1. Kalia LV, Lang AE (2015) Parkinson's disease. Lancet 386 (9996): 896912.

2. DeMaagd G, Philip A (2015) Parkinson's Disease and its management: Part 1: Disease entity, risk factors, pathophysiology, clinical presentation, and diagnosis. PT 40(8): 504-532.

3. Wijarnpreecha K, Chesdachai S, Jaruvongvanich V, Ungprasert P (2018) Hepatitis $\mathrm{C}$ virus infection and risk of Parkinson's disease: a systematic review and meta-analysis. Eur J Gastroentrol Hepatol 30(1): 9-13.

4. Kleine T, Hackler R, Zöfel P (1993) Age-related alterations of the bloodbrain-barrier (bbb) permeability to protein molecules of different size. Z Gerontol 26(4): 256-259.

5. Valiathan R, Ashman M, Asthana D (2016) Effects of ageing on the immune system: infants to elderly. Scand J Immunol 83(4): 255-266.

6. Olsen LK, Dowd E, McKernan DP (2018) A role for viral infections in Parkinson's etiology? Neuronal Signaling 2(2): 1-14.

7. Jang H, Boltz DA, Webster RG, Smeyne RJ (2009) Viral parkinsonism. Biochim Biophys Acta 1792(7): 714-721. 
8. Laskus T, Radkowski M, Adair DM, Wilkinson J, Scheck AC, et al. (2005) Emerging evidence of hepatitis C neuroinvasion. AIDS 19(Suppl 3): 140 144.

9. Forton DM, Allsop JM, Main J, Foster GR, Thomas HC, et al. (2001) Evidence for a cerebral effect of the hepatitis C virus. Lancet 358(9275):38-39.

10. Weissenborn K, Ennen JC, Bokemeyer M, Ahl B, Wurster U, et al. (2006) Monoaminergic neurotransmission is altered in hepatitis $\mathrm{C}$ virus infected patients with chronic fatigue and cognitive impairment. Gut 55(11): 1624-1630.

11. Radkowski M, Wilkinson J, Nowicki M, Adair D, Vargas H, et al. (2002) Search for hepatitis $\mathrm{C}$ virus negative-strand RNA sequences and analysis of viral sequences in the central nervous system: evidence of replication. J Virol 76(2): 600-608.

12. Wilkinson J, Radkowski M, Laskus T (2009) Hepatitis C virus neuroinvasion: identification of infected cells. J Virol 83(3): 1312-1319.

13. Wilkinson J, Radkowski M, Eschbacher JM, Laskus T (2010) Activation of brain macrophages/microglia cells in hepatitis C infection. Gut 59(10): 1394-1400.

14. Lucchese G, Kanduc D (2014) Single amino acid repeats connect viruses to neurodegeneration. Curr Drug Discov Technol 11(3): 214-249.

15. Tsai HH, Liou HH, Muo CH, Lee CZ, Yen RF, et al. (2016) Hepatitis C virus infection as a risk factor for Parkinson disease: A nationwide cohort study. Neurology 86(9): 840-846.

16. Wu WYY, Kang KH, Chen SLS, Chiu SYH, Yen AMF, et al. (2015) Hepatitis C virus infection: a risk factor for Parkinson's disease. J Viral Hepat 22(10): 784-791.

17. Kim JM, Jang ES, Ok K, Oh ES, Kim KJ, et al. (2016) Association between hepatitis C virus infection and Parkinson's disease. Movement Disorders 31(10): 1584-1585.

18. Pakpoor J, Noyce A, Goldacre R, Selkihova M, Mullin S, et al. (2017) Viral hepatitis and Parkinson disease: A national record-linkage study. Neurology 88(17): 1630-1633.

19. Golabi P, Otgonsuren M, Sayiner M, Arsalla A, Gogoll T, et al. (2017) The prevalence of Parkinson Disease among patients with Hepatitis $C$ infection. Ann Hepatol 16(3): 342-348.

20. Arndt T, Guessregen B, Hohl A, Reis J (2005) Determination of serum amantadine by liquid chromatography-tandem mass spectrometry. Clin Chim Acta 359(1-2): 125-131.

21. Yeggoni DP, Manidhar DM, Suresh Reddy C, Subramanyam R (2016) Investigation of binding mechanism of novel 8-substituted coumarin derivatives with human serum albumin and $\alpha$-1-glycoprotein. J Biomol Struct Dyn 34(9): 2023-2036.

22. Gay M, Carato P, Coevoet M, Renault N, Larchanché PE, et al. (2018) New phenylanaline derivatives as modulators of amyloid protein precursor metabolism. Bioorg Med Chem 26(8): 2151-2164.
23. Rehman H, Liu Q, Krishnasamy Y, Shi Z, Ramshesh VK, et al. (2016) The mitochondria-targeted antioxidant Mito $\mathrm{Q}$ attenuates liver fibrosis in mice. Int J Physiol Pathophysiol Pharmacol 8(1): 14-27.

24. Bersano A, Aghemo A, Rumi MG, Ballabio E, Candelise L, et al. (2008) Recovery after L-DOPA treatment in peginterferon and ribavirin induced parkinsonism. Eur J Intern Med 19(5): 370-371.

25. Wangensteen KJ, Krawit EL, Hamill RW, Boyd JT (2016) Parkinsonism in patients with chronic hepatitis $\mathrm{C}$ treated with interferons: Case reports and review of the literature. Clinical Neuropharmacology 39(1): 1-5.

26. Lu CS, Kao WY, Lin JC, Chang PY (2015) Interferon-alpha caused reversible parkinsonism. Clin Neuropharmacol 38(3): 119-120.

27. Kajihara M, Montagnese S, Khanna P, Amodio P, Schapira AH, et al. (2010) Parkinsonism in patients with chronic hepatitis $C$ treated with interferon-alpha2b: a report of two cases. Eur J Gastroenterol Hepatol 22(5): 628-631.

28. Mizoi Y, Kaneko H, Oharazawa A, Kuroiwa H (1997) Parkinsonism in a patient receiving interferon alpha therapy for chronic hepatitis C. Rinsho Shinkeigaku 37(1): 54-56

29. Almeida CM, Galvão Mde L, Ferreira PL, Braga WS (2009) Interferoninduced Parkinsonism in a patient with chronic hepatitis C. Arq Neuropsiquiatr 67(3A): 715-716.

30. Malaguarnera M, Laurino A, Di Fazio I, Pistone G, Castorina M, et al (2001) Neuropsychiatric effects and type of IFN-alpha in chronic hepatitis C. J Interferon Cytokine Res 21(5): 273-278.

31. Atasoy N, Ustundag Y, Konuk N, Atik L (2004) Acute dystonia during pegylated interferon alpha therapy in a case with chronic hepatitis $B$ infection. Clin Neuropharmacol 27(3): 105-107.

32. Raison CL, Demetrashivili M, Capuron L, Miller AH (2005) Neuropsychiatric adverse effects of interferon-alpha: recognition and management. CNS Drugs 19(2): 105-123.

33. Miyagi S, Sekiguchi S, Kawagishi N, Akamatsu Y, Sato A, et al. (2008) Parkinsonism during cyclosporine treatment in liver transplantation: an unusual case report. Transplant Proc 40(8): 2823-2824.

34. Chen HH, Liu PF, Tsai HH, Yen RF, Liou HH (2016) Re: Wangensteen et al. of a letter on 'Hepatitic C virus infection: a risk factor for Parkinson's disease.' J Viral Hepat 23(7): 560.

35. Spengler U (2018) Direct antiviral agents (DAAs) - A new age in the treatment of hepatitis C virus infection. Pharmacol Ther 183: 118-126.

36. Abushouk AI, El-Husseny MWA, Magdy M, Ismail A, Attia A, et al. (2017) Evidence for association between hepatitis C virus and Parkinson's disease. Neurol Sci 38(11): 1913-1920.

37. Hirsch EC, Hunot S (2009) Neuroinflammation in Parkinson's disease: a target for neuroprotection? Lancet Neurol 8(4): 382-397.
Creative Commons Attribution 4.0

International License

For possible submissions Click Here

\section{Submit Article}

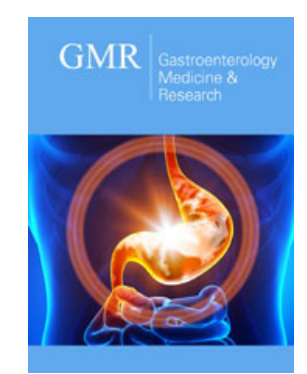

\section{Gastroenterology Medicine \& Research}

\section{Benefits of Publishing with us}

- High-level peer review and editorial services

- Freely accessible online immediately upon publication

- Authors retain the copyright to their work

- Licensing it under a Creative Commons license

- Visibility through different online platforms 